\title{
Mass dependence of short- range correlations in nuclei and the EMC effect
}

\author{
Wim Cosyn ${ }^{1}$, a , Maarten Vanhalst ${ }^{1}$, and Jan Ryckebusch ${ }^{1}$ \\ ${ }^{1}$ Department of Physics and Astronomy, \\ Ghent University, Proeftuinstraat 86, B-9000 Gent, Belgium
}

\begin{abstract}
We sketch an approximate method to quantify the number of correlated pairs in any nucleus $A$. It is based on counting independent-particle model (IPM) nucleonnucleon pairs in a relative $S$-state with no radial excitation. We show that IPM pairs with those quantum numbers are most prone to short-range correlations and are at the origin of the high-momentum tail of the nuclear momentum distributions. Our method allows to compute the $a_{2}$ ratios extracted from inclusive electron scattering. Furthermore, our results reproduce the observed linear correlation between the number of correlated pairs and the magnitude of the EMC effect. We show that the width of the pair center-ofmass distribution in exclusive two-nucleon knockout yields information on the quantum numbers of the pairs.
\end{abstract}

One of the striking features of the nucleon-nucleon potential is the appearance of a huge repulsive core in the potential for inter-nucleon distances of $\lesssim 1 \mathrm{fm}$. This is a reflection of the finite size of the nucleon. The hard core introduces short-range correlations (SRC) in the nuclear quantum many-body system. These cause high-density and high-momentum fluctuations that are reflected in the fat tails of the one-body momentum distribution. Recent theoretical and experimental efforts have identified proton-neutron pairs as the dominant contribution to SRC, an effect which can be attributed to the tensor part of the nuclear force (for recent reviews see [1,2]). Somewhat surprisingly, a very nice linear correlation was also observed between the magnitude of the EMC (European Muon Collaboration) effect for a nucleus $A$ and the ratios of inclusive electron scattering from nucleus $A$ relative to the deuteron (taken as a measure for the amount of correlated pairs in a nucleus) [3, 4]. This might indicate that both phenomena share a common origin, being caused by high-density and/or highvirtuality fluctuations in the nucleus. Here, high-virtuality refers to high-momentum bound nucleons, consequently far off the mass shell.

In Refs. [5-7], we have introduced an approximate method to quantify the number of correlated pairs in any nucleus. We start from the well-known approach where a symmetrical correlation operator $\widehat{\mathcal{G}}$ is used to transform a Slater determinant of independent particle model (IPM) single-particle wave functions $\Psi_{A}^{\mathrm{IPM}}$ into a realistic wave function $\Psi_{A}[8]$ :

$$
\left|\Psi_{A}\right\rangle=\frac{1}{\sqrt{\left\langle\Psi_{A}^{I P M}\left|\widehat{\mathcal{G}}^{\dagger} \widehat{\mathcal{G}}\right| \Psi_{A}^{I P M}\right\rangle}} \widehat{\mathcal{G}}\left|\Psi_{A}^{\mathrm{IPM}}\right\rangle .
$$

\footnotetext{
ae-mail: wim.cosyn@ugent.be
}

This is an Open Access article distributed under the terms of the Creative Commons Attribution License 2.0, which permits unrestricted use, distribution, and reproduction in any medium, provided the original work is properly cited. 
The operator $\widehat{\mathcal{G}}$ is complicated but as far as the SRC are concerned, it is dominated by the central, tensor and spin-isospin correlations $[9,10]$. We then exploit the short-ranged nature of the dominant contributions in the following way. We start from a harmonic oscillator (HO) basis for the Slater determinant $\Psi_{A}^{\mathrm{IPM}}$. This does not impose restrictions on the choice of IPM model as any wave function can be expanded in the HO basis. Next, for the two-particle wave function, we perform a transformation to relative and center-of-mass (c.o.m.) coordinates using Talmi-Moshinsky brackets [11]. This allows us to identify the contributions of all relative $(n l)$ and c.o.m. $(N L)$ HO quantum numbers to the twoparticle wave functions $[5,6]$. As the typical central and tensor correlation operators in Eq. (1) project on pairs with a substantial close-proximity $(r \approx 0)$ probability, we identify the $\operatorname{IPM}(n=0, l=0)$ nucleon pairs as those parts of $\Psi_{A}^{\mathrm{IPM}}$ prone to SRC. The validity of this assumption is nicely illustrated in Fig. 1, where we show the different $(n, l)$ contributions to the two-body momentum distribution in ${ }^{56} \mathrm{Fe}$ as computed in a lowest order cluster approximation of Eq. (1). One clearly observes that the SRC generated high-momentum tail in the momentum distribution is dominated by the contribution from correlation operators acting on IPM pairs with $(n=0, l=0)$ relative quantum numbers. We wish to stress that the high-momentum tails in the momentum distributions can have very different quantum numbers than $(n=0, l=0)$ through the operation of the correlation operator $\mathcal{G}$. The most obvious example is the dynamical generation of the deuteron D-wave $(l=2)$ as the tensor part of $\widehat{\mathcal{G}}$ acts on the "IPM" S-wave $(l=0)$ component of the wave function.

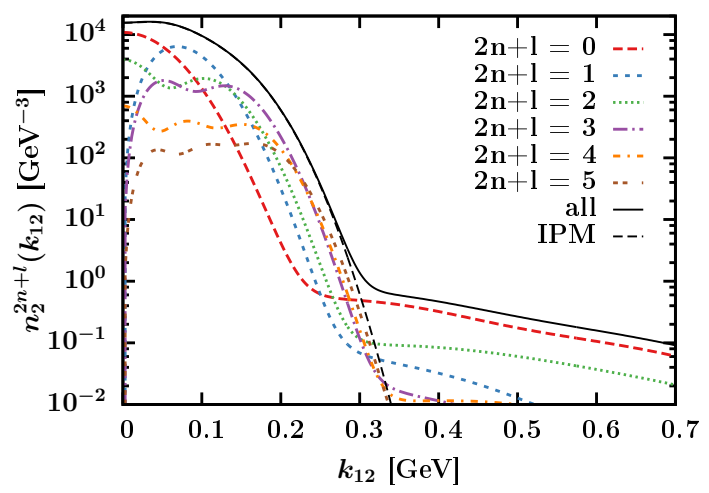

Figure 1: (Color online) Momentum dependence of the relative two-body momentum distribution $n_{2}$ (solid black curve) in function of the relative momentum $k_{12}$ of the two nucleons, as computed in the lowest-order cluster expansion for ${ }^{56} \mathrm{Fe}$. The black dashed curve is the IPM prediction. The other lines show the different contributions $n_{2}^{2 n+l}$ to $n_{2}$ from the correlation operators acting on two-nucleon states with various $n l$.

The mass dependence of nuclear SRC has experimentally been quantified in inclusive electron scattering (for an overview of the world data see [12]). The ratio of the inclusive cross section at moderate $Q^{2}$ on a nucleus $A$ to the deuteron shows a scaling plateau at values of Bjorken $x_{B}$ of $1.5 \lesssim x_{B} \lesssim 2$. The value of the measured ratio rescaled by $\frac{2}{A}$ (a quantity denoted by $a_{2}(A)$ ) can be interpreted as a measure of the amount of correlated pairs in $A$ relative to the deuteron. We compare in Fig. 2a the experimental values of these $a_{2}$ in superratios to ${ }^{4} \mathrm{He}$ with our calculations. The kinematics in the inclusive experiments probe initial nucleon momenta in the range of 300-500 MeV, where the tensor correlation function forms the dominant contribution to the high-momentum tail. Thereby, in our calculations we compute the $a_{2}(A)$ by counting the close-proximity $(n=0, l=0) n p$ pairs in a 
spin triplet $(S=1)$ state. One can observe that the predictions are in acceptable agreement with the slope and magnitude of the measured $A$-dependence data for light and medium nuclei, but tend to overestimate the measured values at high $A$. No corrections accounting for the c.o.m. motion or finalstate interactions of the pairs in the nucleus $A$ were applied to the calculations in Fig. 2a, providing a possible explanation for this overestimation. It is also obvious that the scaling with $A$ of $a_{2}(A)$ is a lot softer than one would expect from the naive counting of all $n p$ pairs, also depicted in Fig. $2 \mathrm{a}$.

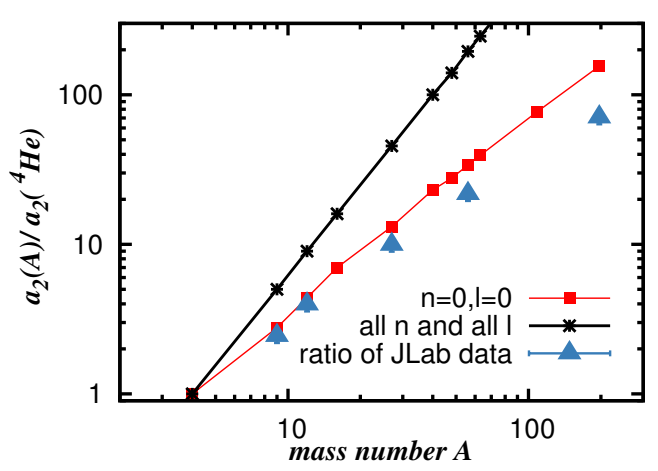

(a)

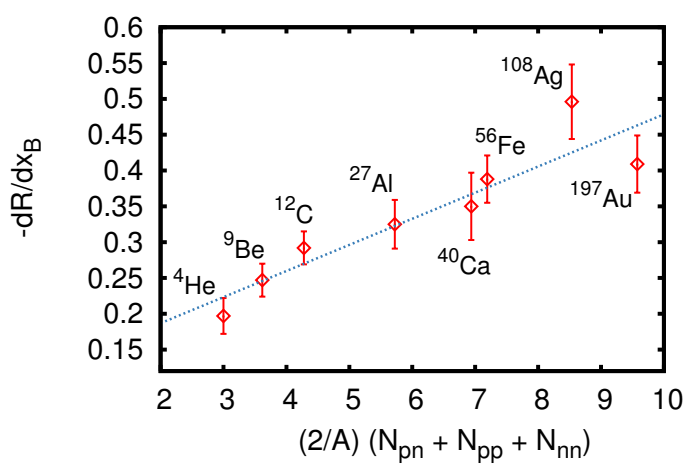

(b)

Figure 2: (Color online) (a) Mass dependence of the $a_{2}$ relative to ${ }^{4} \mathrm{He}$. The black crosses are the predictions given that all relative quantum numbers equally contribute. For the red squares only $p n$ pairs in a triplet spin state and with $n=0, l=0$ are counted. The data are from Ref. [12]. The curves are guide to the eyes. (b) The magnitude of the EMC effect versus the computed number of correlated $N N$ pairs per nucleon. The EMC data are from the analysis presented in Ref. [12].

Given the experimentally established linear correlation between the size of the EMC effect and the amount of correlated pairs in a nucleus, we want to check if this correlation is also present when we compare our model calculations to EMC data. In Fig. 2b, we display the magnitude of the EMC effect - quantified by means of the slope $-d R / d x_{B}$ of the EMC ratio for Bjorken $0.35<x_{B}<0.7$ [13] - versus our predictions for the probability for $N N$ SRC per nucleon relative to the deuteron. Here, contrary to the $a_{2}$ ratios in Fig. $2 \mathrm{a}$, we compare to the combined number of $p p, n p$ and $n n$ pairs. This is motivated by the partonic nature of the deep-inelastic scattering process (all pairs can contribute equally), whereas the inclusive scattering is dominated by the tensor-correlated triplet-spin protonneutron pairs. We stress that the numbers which one finds on the $x$ axis of Fig. 2a are the results of parameter-free calculations. A nice linear relationship can be observed between the quantity which we propose as a per nucleon measure for the magnitude of the SRC and the magnitude of the EMC effect. If the observed trends apply to the whole nuclear mass range, we could predict the size of the EMC effect for any nucleus $A$.

So far, in Fig. 2, we have compared our model calculations to quantities extracted from inclusive electron scattering experiments. To study the precise nature of nuclear SRC, the exclusive $A\left(e, e^{\prime} N N\right)$ reaction provides more opportunities than inclusive measurements, but is of course more demanding to measure. Similarly to the scaling with the distorted one-body momentum distribution for the quasi-elastic $A\left(e, e^{\prime} N\right)$ cross section, it can be shown that under kinematic conditions probing correlated nucleon-nucleon pairs, the $A\left(e, e^{\prime} N N\right)$ cross section scales with the distorted c.o.m. momentum distribution of close-proximity pairs. When comparing the width of the computed c.o.m. momentum distribution restricted to the $(n=0, l=0)$ pairs, with the width of the distribution for all pairs, one 
Table 1: The width of the ${ }^{12} \mathrm{C}$ c.m. distribution for pp pairs with relative orbital momentum $l$.

\begin{tabular}{ccccc}
\hline & $l=0$ & $l=1$ & $l=2$ & all $l$ \\
\hline$\sigma[\mathrm{MeV}]$ & 154 & 135 & 121 & 140 \\
\hline
\end{tabular}

observes a significant difference between the two. The numbers for the widths of the c.o.m. distributions for several relative $l$ quantum numbers in ${ }^{12} \mathrm{C}$ are listed in Table 1. The width of the c.o.m. distribution for close-proximity $l=0$ pairs is $14 \mathrm{MeV}$ wider than the one for the distribution which does not impose restrictions on the $l$.

To conclude, we have shown that the amount of correlated pairs in any nucleus can be approximately quantified by counting the number of relative $(n=0, l=0)$ pairs in the IPM wave function. Our calculations for the $a_{2}$ ratios are consistent with the data for light and medium nuclei, but tend to overestimate these at high $A$. We observe a nice correlation between the computed number of correlated pairs and the measured magnitude of the EMC effect. We have shown that the width of the c.o.m. momentum distribution differs significantly between all possible and correlated nucleonnucleon pairs, the latter being $14 \mathrm{MeV}$ wider in ${ }^{12} \mathrm{C}$. This is a quantity that can be accessed in exclusive $A\left(e, e^{\prime} N N\right)$ reactions at moderate $Q^{2}$.

\section{Acknowledgements}

This work is supported by the Research Foundation Flanders (FWO-Flanders) and by the Interuniversity Attraction Poles Programme initiated by the Belgian Science Policy Office. The computational resources (Stevin Supercomputer Infrastructure) and services used in this work were provided by Ghent University, the Hercules Foundation and the Flemish Government - department EWI.

\section{References}

[1] J. Arrington, D. Higinbotham, G. Rosner, M. Sargsian, Prog. Part. Nucl. Phys. 67, 898 (2012)

[2] L. Frankfurt, M. Sargsian, M. Strikman, Int. J. Mod. Phys. A23, 2991 (2008)

[3] L.B. Weinstein, E. Piasetzky, D.W. Higinbotham, J. Gomez, O. Hen, R. Shneor, Phys. Rev. Lett. 106, 052301 (2011)

[4] O. Hen, D. Higinbotham, G. Miller, E. Piasetzky, L. Weinstein, Int. J. Mod. Phys. E. 22, 1330017 (2013)

[5] M. Vanhalst, W. Cosyn, J. Ryckebusch, Phys. Rev. C84, 031302 (2011)

[6] M. Vanhalst, J. Ryckebusch, W. Cosyn, Phys.Rev. C86, 044619 (2012)

[7] M. Vanhalst, J. Ryckebusch, W. Cosyn (2012), 1210.6175

[8] S.C. Pieper, R.B. Wiringa, V. Pandharipande, Phys. Rev. C46, 1741 (1992)

[9] S. Janssen, J. Ryckebusch, W. Van Nespen, D. Debruyne, Nucl. Phys. A 672, 285 (2000)

[10] J. Ryckebusch et al., Nucl. Phys. A 624, 581 (1997)

[11] M. Moshinsky, Y. Smirnov, The harmonic oscillator in modern physics (Harwood Academic Publishers,Amsterdam, 1996)

[12] O. Hen, E. Piasetzky, L. Weinstein, Phys. Rev. C85, 047301 (2012)

[13] J. Seely, A. Daniel, D. Gaskell, J. Arrington, N. Fomin et al., Phys.Rev.Lett. 103, 202301 (2009) 